$\$$ Research Square
Preprints are preliminary reports that have not undergone peer review.

They should not be considered conclusive, used to inform clinical practice, or referenced by the media as validated information.

\title{
A Research of STEAP1 on the Biological Behavior of Gastric Cancer
}

\section{Zhe Zhang}

China Medical University

Huimian Xu ( $\nabla$ xuhuimian123@163.com )

Department of Oncology surgery, the First Affiliated Hospital of China Medical University, Shenyang, China https://orcid.org/0000-0002-1177-4445

Primary research

Keywords: STEAP1, Gastric cancer, Proliferation, migration, invasion

Posted Date: August 21st, 2020

DOl: https://doi.org/10.21203/rs.3.rs-59451/v1

License: (1) This work is licensed under a Creative Commons Attribution 4.0 International License. Read Full License 


\section{Abstract}

Introduction:Six-Transmembrane Epithelial Antigene of the Prostate 1 (STEAP1) is associated with the occurrence and development of cancer. This study aimed to clarify the role of STEAP1 in gastric cancer tumor growth and metastasis, as well as its molecular mechanism of action.

Methods \Statistical methods were used for clinical data analysis. Protein expression was detected using immunohistochemistry $(\mathrm{IHC})$. The mRNA and protein expression in the cell cultures were detected using reverse transcription-polymerase chain reaction(RT-PCR) and western blot analysis. Overexpression and silencing models were constructed using plasmid and lentivirus transfection. To detect cell proliferation in vitro, Cell Counting Kit-8(CCK-8), flow cytometry, and colony formation assays were used; transwell and wound healing assays were used to detect cell migration and invasion; For in vivo experiments, nude $\mathrm{BALB} / \mathrm{c}$ mice were used for detecting subcutaneous tumorigenesis and intraperitoneal implantation.

Results $₫$ We found STEAP1 was overexpressed in gastric cancer tissues and cell lines. Single factor and Cox analyses showed that STEAP1 gene expression level correlated with poor prognosis. Upregulation of STEAP1 increased cell proliferation, migration, and invasion, which decreased after STEAP1 was knocked down. These changes were achieved via the activation of the AKT/Fox01 pathway and epithelialmesenchymal transformation (EMT). The in vivo animal experiments showed that STEAP1 knock down, resulted in a decrease in the subcutaneous tumor and peritoneal tumor formation.

Conclusions囚STEAP1 was overexpressed in gastric cancer and closely connected with OS. STEAP1 can regulate the cell cycle via the Akt/Fox01 pathway to influence cell proliferation. STEAP1 may affect cell migration and invasion via EMT induction.

\section{Introduction}

Gastric cancer is a common kind of malignant tumor that seriously affects people's health.[1] According to the data from the World health Organization (WHO), in contrast to the United States, Australia and New Zealand, China, Japan, and Chile have higher incidence areas of gastric cancer.[2] In China, gastric cancer is the second cause of cancer-related deaths, with 679000 new cases and 498000 deaths.[3] Gastric cancer is predisposed to occur in individuals aged between 50 and 70 years. However, in recent years, it has shown a "younger" trend.[4-9] Therefore, it is essential to find new tumor markers to predict the risk of gastric cancer progression.

Tumor development involves many factors, which are controlled by many genes, including prostate transmembrane epithelial antigen. Six-Transmembrane Epithelial Antigene of the Prostate (STEAP) was found as a prostate-specific cell surface antigen using suppression subtractive hybridization technique for the first time[10,11]. STEAP is highly expressed in spontaneous transgenic mouse prostate cancer models and human prostate cancer. In addition, it is also expressed in the pancreas, ovary, gastrointestinal tract, cervix, testis, bladder, Ewing sarcoma, and melanoma cells[10, 12]. There are four members in the STEAP protein family, STEAP1-4. The main focus of our study is STEAP1. 
Gene STEAP1 is located in the 7q21.13 region of the human chromosome; it is $10.4 \mathrm{~kb}$ long and contains four introns and five exons. The transcription of the gene STEAP1 can produce two different kinds of mRNAs: a $1.4 \mathrm{~kb}$ and a $4 \mathrm{~kb}$ mRNAs. However, only the $1.4 \mathrm{~kb}$ mRNA can be processed into a mature protein, which contains 339 amino acids with molecular weight of $36 \mathrm{KD} .[10,12]$, while the $4 \mathrm{~kb} \mathrm{mRNA}$ contains a $2399 \mathrm{BP}$ large intron, which is not translated into a mature protein[13]. Data has shown that gene STEAP1 is closely related to communication between the adjacent cells, and it seemed to be beneficial for the occurrence and development of tumors.[14] Its structural prediction and the location at the cell-cell contacts indicated that gene STEAP1 product may be a transporter or channel.[10, 15] Some previous research on different kinds of cancer have found that STEAP1 was observed in tumor tissue but not in normal tissue. The expression of STEAP1 was closely related to the malignant phenotype of cancer cells.[13, 16-20] However, in Lee's study, they found no correlation between expression of STEAP1 and the clinicopathological factors[21]. These conflicting results indicated that the roles of STEAP1 were varied depending on different cancer types. In our study, we will discuss the influence of STEAP1 on the proliferation, invasion, and inflammatory reactions in gastric cancer.

\section{Materials And Methods}

\section{Clinical samples}

212 samples of gastric cancer tissue and 60 samples of adjacent normal gastric tissue from patients were obtained from the First Affiliated Hospital of China Medical University from 2003 to 2010. None of the patients received preoperative chemotherapy or radiotherapy, and all of them were proven to have gastric cancer by pathology. The cancer tissue was fixed with formalin and preserved in paraffin. All pathological data were complete, and the postoperative follow-up was sufficient. All patients were approved by the ethics committee of China Medical University to participate in the study and provided written informed consent.

\section{Immunohistochemistry of human gastric cancer}

To fix gastric cancer tissue samples, $10 \%$ formalin was used, then the tissue was paraffin-embedded and cut into $4-\mu \mathrm{m}$ slices. The xylene and alcohol were used for dewaxing and rehydrating. Endogenous peroxidase activity was blocked by using hydrogen peroxide (30\%), the citrate buffer $(\mathrm{pH} 6.0)$ was used to boil the the sections fro 3 minutes in a pressure cooker. Next, normal goat serum was used for incubating the sections to reduce the nonspecific binding. Finally, the tissue sections were incubated $\left(4^{\circ} \mathrm{C}, 12 \mathrm{~h}\right)$ with anti-STEAP1 antibody (1:200 dilution, B-4, SC-271872, Santa Cruz, USA). Enzyme labeled anti mouse / rabbit IgG polymer was used for secondary antibody. (Mai Xin Biological company,Fuzhou,China). Finally, the sections were stained with diaminobezidin (DAB) for $60 \mathrm{sec}$, stained with hematoxylin for 2 min and sealed with neutral resin. Fluorescence photographic microscope was used for obtaining images(Nikon,Japan).Two pathologists examined all tumor slides randomly. We evaluated STEAP1 staining intensity as follows: scored 0 (negative), 1 (weakly negative), 2 (weak positive), and 3 (strong positive). The percentage scores of positive cells per single field vision were as follows: scored 1 ( $0-$ 
25\%), 2 (26-50\%), 3 (51-75\%), and 4 (76-100\%). We multiplied the two scores above and obtained a final score ranging from 0 to 12 . Tumor samples with a score $<6$ were considered as negative expression; on the contrary, the score $\geq 6$ was considered as positive expression.

\section{Animals}

Twenty-four BALB/c nude female mice were purchased from Beijing Vital River Laboratory Animal Technology Co., Ltd. The mice were raised in the animal experimental center of of China Medical University. The twenty-four nude mice were randomly divided into four groups. Two groups were used for hypodermic injection, and the other two groups were used for intraperitoneally injected .In the subcutaneous tumorigenesis experiment, $3 \times 106$ SGC-7901 NC cells per mouse were injected in NC group,and 3*106 SGC-7901 sh-STEAP1 cells per mouse were injected in sh-STEAP1 group .Similarly, in the experiment of intraperitoneal tumorigenesis, $3^{*} 106$ SGC-7901 NC cells per mouse were injected in NC group,and $3 \times 106$ SGC-7901 sh-STEAP1 cells per mouse were injected in sh-STEAP1 group .The animals experiments primary antibodies were used as follows. STEAP1 (Ab207914,1:200) was purchased from Abcam. Ki-67(GB111141,1:500),C-CASPASE3(GB13436,1:200) were provided by Wuhan Servicebio technology Co., Ltd. IL1ß(16806-1-AP, 1:200),IL6 (66146-1-AP, 1:200) were purchased from Proteintech Group, Wuhan, China. Secondary antibodies were used as follows, the goat anti-rabbit (GB23303, 1:200)and anti-mouse IgG (GB23301, 1:200) secondary antibodies were provided by Wuhan Servicebio technology Co., Ltd. All animal experimental steps were approved by the Animal Research Committee of China Medical University.

\section{Cell culture}

GES-1, a normal gastric mucosa cell line was obtained from Nanjing Cobioer Biotechnology Co., Ltd. The gastric cancer cell lines MGC-803 and SGC-7901 were obtained from the Chinese Academy of Sciences. AGS was purchased from the American Type Culture Collection (ATCC, Manassas, VA, USA). MGC-803, SGC-7901 and GES-1 cells were cultured in DMEM containing 10\% FBS. AGS line was cultured in DMEMF12 medium containing $10 \%$ FBS. All cells were cultured at $37^{\circ} \mathrm{C}$ in a $5 \% \mathrm{CO} 2$ incubator.

\section{Cell transfection}

ShRNA lentivirus was purchased from Shanghai Genechem Co., Ltd. The NC group insertion sequence was TTCTCCGAACGTGTCACGT. There were three shRNA sequences used (shRNA1:

CCAACTTCATAATGGAACCAA; shRNA2: CAGCACACACAGGAACTCTTT; and shRNA3:

AAGCTAGGAATTGTTTCCCTT). The STEAP1 containing CDNA plasmid and Flag empty plasmid were purchased from Beijing SinoBiological Co., Ltd. Lipofectamine 3000 reagent (Thermo Fisher Scientific, Inc.) was used for plasmid transfection. The cells were harvested $48 \mathrm{~h}$ after transfection. Western blot analysis and RT-PCR were used to check the transfection efficiency.

\section{RT-PCR}


TRIzol reagent was used to extracted total RNA. The reverse transcription kit PrimeScript RT was purchased from Takara. The primer designs were provided by Huada Gene Co., and the sequences were shown in Table 1.

Table 1

RNA primer sequence details

\begin{tabular}{|c|c|c|}
\hline Genes & Forward/ Reverse & Sequences \\
\hline \multirow[t]{2}{*}{ GAPDH } & Forward & 5'-GTCTCCTCTGACTTCAACAGCG-3' \\
\hline & Reverse & 5'-ACCACCCTGTTGCTGTAGCCAA-3' \\
\hline \multirow[t]{2}{*}{$\beta$-actin } & Forward & 5'-CACCATTGGCAATGAGCGGTTC-3' \\
\hline & Reverse & 5'-AGGTCTTTGCGGATGTCCACGT-3' \\
\hline \multirow[t]{2}{*}{ CCND1 } & Forward & 5'-TCTACACCGACAACTCCATCC-3' \\
\hline & Reverse & 5'-TCTGGCATTTTGGAGAGGAAGTG-3' \\
\hline \multirow[t]{2}{*}{ P27 } & Forward & 5'-ATAAGGAAGCGACCTGCAACCG-3' \\
\hline & Reverse & 5'-TTCTTGGGCGTCTGCTCCACAG-3' \\
\hline \multirow[t]{2}{*}{ CDK4 } & Forward & 5'-CTCGTGCTGATGCTACTGAGGA-3' \\
\hline & Reverse & 5'-GGTCGGCGCAGTTGGGCTCC-3' \\
\hline \multirow[t]{2}{*}{ E-cadherin, } & Forward & 5'-GCCTCCTGAAAAGAGAGTGGAAG-3' \\
\hline & Reverse & 5'-TGGCAGTGTCTCTCCAAATCCG-3' \\
\hline \multirow[t]{2}{*}{ N-cadherin } & Forward & 5'-CCTCCAGAGTTTACTGCCATGAC-3' \\
\hline & Reverse & 5'-GTAGGATCTCCGCCACTGATTC-3' \\
\hline \multirow[t]{2}{*}{ MMP-2 } & Forward & 5'-AGCGAGTGGATGCCGCCTTTAA-3' \\
\hline & Reverse & 5'-CATTCCAGGCATCTGCGATGAG-3' \\
\hline \multirow[t]{2}{*}{ MMP9 } & Forward & 5'-GCCACTACTGTGCCTTTGAGTC-3' \\
\hline & Reverse & 5'-CCCTCAGAGAATCGCCAGTACT-3' \\
\hline
\end{tabular}

\section{Western blot analysis}

We used RIPA lysis buffer(purchased from Beyotime company) to lysis cells and obtain proteins. Proteins were separated using sodium dodecyl sulfate-polyacrylamide gel electrophoresis (SDS-PAGE, 8\%). After transferring to a polyvinylidene fluoride (PVDF) membrane (Millipore, Billerica, MA, USA), the membranes were incubated overnight at $4{ }^{\circ} \mathrm{C}$ with antibodies against STEAP1 (SC-271872, 1:400) from Santa Cruz, USA. The other antibodies used are as follows. $\beta$-actin (60008-1-Ig, 1:10000)was purchased from Proteintech Group, Wuhan, China. P27 (3686, 1:1000), CDK4 (2546, 1:1000), AKT (4691, 1:1000), P-AKT 
(4060, Ser473, 1:2000), Fox01 (2880, 1:1000), P-Fox01 (9464, 1:2000), E-cadherin (14472, 1:1000), Ncadherin (13116, 1:1000), Vimentin (5741, 1:1000), MMP-2 (4022, 1:1000), MMP9 (13667, 1:1000), were purchased from Cell Signaling Technology, USA. Then the goat anti-rabbit and anti-mouse IgG secondary antibodies were used to incubate the membrane at room temperature for $60 \mathrm{~min}$. Finally, the ECL was used to visualize and detect the proteins by using Biolmaging Systems (UVP Inc., Upland, CA, USA).

\section{Cell Counting Kit-8 (CCK-8) assay}

SGC-7901 and MGC-803 cells were transfected with an empty vector, STEAP1 plasmid, negative control virus, or sh-STEAP1 virus. Cells were seeded at 3000 per well into a 96-well plate, and CCK-8 solution (Beyotime, Shanghai, China) was added into every well at 24 h, 48 h, $72 \mathrm{~h}$ and $96 \mathrm{~h}$. Each group of cells was set with three auxiliary holes, repeat the experiment three times. A microplate reader was used to measure the absorbance values and estimate the cell proliferation rates.

\section{Colony formation assay}

For the colony formation assay, SGC-7901 and MGC-803 cells were transfected with plasmid or shRNA for $36 \mathrm{~h}$ and plated into 6 -well cell plates ( $1000 \mathrm{cells} /$ well). The cells were cultured in a $37^{\circ} \mathrm{C}$ incubator for 2-3 weeks, fixed with alcohol for $30 \mathrm{~min}$, and stained with Trypan Blue for $20 \mathrm{~min}$ at room temperature. The colonies with more than 50 cells were counted. Finally, an HD camera was used to obtain the images. The experiment was repeated three times.

\section{Flow cytometry}

We used a flow cytometry assay to detect the cell cycle stage. SGC-7901 and MGC-803 cells were transfected with plasmid or shRNA and plated into 6-well plates $\left(1 \times 10^{5}\right.$ cells/well). After $24 \mathrm{~h}$, the cells were harvested using $0.25 \%$ trypsin in $1.5 \mathrm{ml}$ Eppendorf tubes. Then, the cells were stained with propidium iodide ( $\mathrm{PI}, 500 \mu$ tube, KeyGEN, Nanjing, China) at $37^{\circ} \mathrm{C}$ in the dark for $30 \mathrm{~min}$. Finally, the cells were analyzed using a FACSCalibur flow cytometer (Becton Dickinson, USA). The experiment was repeated three times.

\section{Transwell assay}

Cell migration experiments were performed using a 24-well transwell chamber with a pore size of $8 \mu \mathrm{m}$ (Costar). A total of $5 \times 10^{4}$ cells in serum-free DMEM were placed in the upper chamber, and DMEM with $10 \%$ FBS was added to the lower chamber. After more than 10 hours, the migration experiment was terminated, and the cells were observed in the medium below. Then, the cells on the membrane in the bottom chamber were fixed with $75 \%$ alcohol for 30 min and stained with Trypan Blue at room temperature for $20 \mathrm{~min}$. Images were obtained using an inverted microscope. In addition, the transwell chamber was also used for cell invasion experiments. For these experiments, in addition to the above steps, Matrigel (1:9 dilution, BD Bioscience) was added to the upper chamber to observe the change in cell invasion ability.The experiment was repeated three times.

\section{Wound healing assay}


A wound healing assay was used to observe the migration of cells. In this study, $1 \times 10^{5}$ cells were seeded into 6-well plates for every group. After the cells had covered the entire plate, a pipette tip was used to make a scratch in the cell monolayer, and phosphate buffer saline (PBS) was used to wash the floating cells three times. Subsequently, we used serum-free DMEM instead of the former medium. Finally, an inverted microscope (Olympus, Japan) was used to take images at $0 \mathrm{~h}$ and $96 \mathrm{~h}$. The difference in scratch distance between the two phases can reflect the difference in the cell migration ability.The experiment was repeated three times.

\section{Statistical analysis}

GraphPad Prism 7.0 was used for image editing. SPSS 21.0 statistical software was used for data analysis.The data of three repeated experiments were input to analysis and expressed as the means \pm SEMs. The chi-square test was used to examine possible correlations between STEAP1 expression and clinicopathological factors. Survival rates were calculated using Kaplan-Meier analysis. The log-rank test was used for single-factor analysis. Cox risk proportion model was used for multi-factor analysis, and a value of $P<0.05$ was considered statistically significant.

\section{Results}

\section{STEAP1 was highly expressed in gastric cancer tissue and closely connected with OS.}

The GEPIA database showed that the STEAP1 gene was more highly expressed in gastric cancer than in the normal tissue (Fig. 1a). We detected 212 cases of gastric cancer tissues and 60 cases of paracancerous tissues using IHC and scored them. The results showed that STEAP1 was highly expressed in cancer tissues and was mainly localized to the membrane and cytoplasm in cells (Fig. 1b). The positive expression rate of STEAP1 was $54.7 \%$ (116/212). However, it was expressed at low levels or was negative in paracancerous tissues (Fig. 1C). The 5-year OS in the high expression group was 25.9\%, which was significantly lower than $60.7 \%$ in patients with low expression group $(P<0.001$, Fig. d). Figure 1e shows the detailed score in 212 cases of tumor tissues and in 60 cases of paracancerous tissues. The scores between the two groups were significantly different $(P<0.001)$. In addition, 60 matched tissues were scored, and the details are shown in Fig. $1 \mathrm{f}(P<0.001)$. The subsequent study of the data of 212 clinical cases showed that the factors that affected the prognosis in patients included tumor location $(P=0.029)$, tumor size $(P=0.012)$, Borrmann type $(P=0.019)$, STEAP1 expression $(P<$ $0.001), \mathrm{N}$ stage $(P<0.001)$, T stage $(P<0.001)$, and distant metastasis $(P=0.005)$ (Table 2$)$. Cox multifactor analysis showed that the independent factors influencing the prognosis of patients included STEAP1 expression $(P<0.001)$, T stage $(P=0.005)$, and $\mathrm{N}$ stage $(P<0.001)$ (Table 3$)$. In the study of the relationship between the expression of STEAP1 and clinicopathological factors, high expression of STEAP1 was closely related to Borrmann type $(P=0.009)$ and N stage $(P<0.001)$ (Table 4$)$. 
Table 2

The clinicopathological data of the 212 patients

\begin{tabular}{|c|c|c|c|}
\hline Clinicopathological factors & $\begin{array}{l}\text { Patients (\%) } \\
212(100))\end{array}$ & $\begin{array}{l}\text { 5-year OS } \\
(100 \%)\end{array}$ & $P$-value \\
\hline Sex & & & 0.637 \\
\hline Male & $161(75.9)$ & 42.9 & \\
\hline Female & $51(24.1)$ & 39.2 & \\
\hline Age (years) & & & 0.675 \\
\hline$\leq 60$ & $103(48.6)$ & 42.7 & \\
\hline$>60$ & $109(51.4)$ & 41.3 & \\
\hline Location & & & 0.029 \\
\hline Upper & $26(12.3)$ & 38.5 & \\
\hline Middle & $29(13.7)$ & 44.8 & \\
\hline Lower & $143(67.5)$ & 44.8 & \\
\hline Entire & $14(6.6)$ & 14.3 & \\
\hline Size (cm) & & & 0.012 \\
\hline$\leq 4$ & $55(25.9)$ & 56.4 & \\
\hline$>4$ & $157(74.1)$ & 36.9 & \\
\hline Borrmann Type & & & 0.019 \\
\hline Borrmann 1 & $8(3.8)$ & 50.0 & \\
\hline Borrmann 2 & $14(6.6)$ & 78.6 & \\
\hline Borrmann 3 & 185(87.3) & 39.5 & \\
\hline Borrmann 4 & $5(2.3)$ & 20.0 & \\
\hline Differentiation degree* & & & 0.472 \\
\hline Differentiated & $102(48.1)$ & 39.2 & \\
\hline Undifferentiated & $110(51.9)$ & 44.5 & \\
\hline STEAP1 expression status & & & 0.000 \\
\hline Low & $96(45.3)$ & 60.7 & \\
\hline
\end{tabular}

* High and medium differentiated tubular adenocarcinoma and papillary adenocarcinoma were regarded as differentiated types; mucous adenocarcinoma, signet ring cell carcinoma, low and undifferentiated adenocarcinoma were regarded as undifferentiated types. 


\begin{tabular}{|c|c|c|c|}
\hline Clinicopathological factors & $\begin{array}{l}\text { Patients (\%) } \\
212(100))\end{array}$ & $\begin{array}{l}\text { 5-year OS } \\
(100 \%)\end{array}$ & $P$-value \\
\hline High & $116(54.7)$ & 25.9 & \\
\hline \multicolumn{2}{|l|}{ Lymphovascular invasion } & & 0.052 \\
\hline Negative & 139(65.6) & 61.5 & \\
\hline Positive & $73(34.4)$ & 32.9 & \\
\hline \multicolumn{2}{|l|}{ T staging } & & $<0.001$ \\
\hline T1-2 & $33(15.6)$ & 57.6 & \\
\hline T3 & $34(16.0)$ & 58.8 & \\
\hline T4a & $139(65.6)$ & 35.3 & \\
\hline $\mathrm{T} 4 \mathrm{~b}$ & $6(2.8)$ & 16.7 & \\
\hline \multicolumn{2}{|l|}{$\mathrm{N}$ staging } & & $<0.001$ \\
\hline NO & $51(24.1)$ & 70.6 & \\
\hline N1 & $48(22.6)$ & 45.8 & \\
\hline N2 & $35(16.5)$ & 45.7 & \\
\hline N3a & $50(23.6)$ & 24.0 & \\
\hline N3b & $28(13.2)$ & 10.7 & \\
\hline M0 or M1 & & & 0.005 \\
\hline MO & $201(94.8)$ & 43.3 & \\
\hline M1 & $11(5.2)$ & 10.7 & \\
\hline $\begin{array}{l}\text { * High and medium differen } \\
\text { regarded as differentiated ty } \\
\text { undifferentiated adenocarci }\end{array}$ & $\begin{array}{l}\text { enocarcinom } \\
\text { enocarcinoma } \\
\text { ded as undiff }\end{array}$ & $\begin{array}{l}\text { Ilary adeno } \\
\text { ig cell carci } \\
\text { types. }\end{array}$ & $\begin{array}{l}\text { na were } \\
\text { ow and }\end{array}$ \\
\hline
\end{tabular}


Table 3

Multifactorial analysis

\begin{tabular}{|llll|}
\hline Clinicopathological factors & $\operatorname{Exp}(\mathrm{B})$ & $\mathbf{9 5 \%} \mathrm{Cl}$ & $\boldsymbol{P}$-value \\
\hline Borrmann Type & 0.934 & $0.783-1.114$ & 0.449 \\
\hline Location & 1.131 & $0.700-1.827$ & 0.614 \\
\hline Size $(\mathrm{cm})$ & 0.919 & $0.573-1.473$ & 0.725 \\
\hline STEAP1 expression status & 2.203 & $1.468-3.300$ & $<0.001$ \\
\hline M0 or M1 & 1.329 & $0.652-2.707$ & 0.433 \\
\hline T staging & 1.512 & $1.132-2.020$ & 0.005 \\
\hline N staging & 1.343 & $1.162-1.553$ & $<0.001$ \\
\hline Lymphovascular invasion & 1.214 & $0.838-1.759$ & 0.304 \\
\hline
\end{tabular}


Table 4

\begin{tabular}{|c|c|c|c|}
\hline Clinicopathological factors & Positive (cases) & Negative (cases) & $P$-value \\
\hline Sex & & & 0.100 \\
\hline Male & 78 & 83 & \\
\hline Female & 18 & 33 & \\
\hline Age (years) & & & 0.921 \\
\hline$\leq 60$ & 47 & 56 & \\
\hline$>60$ & 49 & 60 & \\
\hline Location & & & 0.103 \\
\hline Upper & 13 & 13 & \\
\hline Middle & 14 & 15 & \\
\hline Lower & 77 & 66 & \\
\hline Entire & 12 & 2 & \\
\hline Size $(\mathrm{cm})$ & & & 0.510 \\
\hline$\leq 4$ & 27 & 28 & \\
\hline$>4$ & 69 & 88 & \\
\hline Borrmann Type & & & 0.009 \\
\hline Borrmann 1 & 1 & 7 & \\
\hline Borrmann 2 & 4 & 10 & \\
\hline Borrmann 3 & 107 & 78 & \\
\hline Borrmann 4 & 4 & 1 & \\
\hline Differentiation degree* & & & 0.823 \\
\hline Differentiated & 47 & 55 & \\
\hline Undifferentiated & 49 & 61 & \\
\hline Lymphovascular invasion & & & 0.142 \\
\hline Negative & 71 & 68 & \\
\hline Positive & 45 & 28 & \\
\hline
\end{tabular}

* High and medium differentiated tubular adenocarcinoma and papillary adenocarcinoma were regarded as differentiated types; mucous adenocarcinoma, signet ring cell carcinoma, low and undifferentiated adenocarcinoma were regarded as undifferentiated types. 


\begin{tabular}{|llll|}
\hline Clinicopathological factors & Positive (cases) & Negative (cases) & P-value \\
\hline T staging & & & 0.619 \\
\hline T1-2 & 18 & 15 & \\
\hline T3 & 16 & 18 & \\
\hline T4a & 60 & 79 & \\
\hline T4b & 2 & 4 & \\
\hline N staging & & & \\
\hline N0 & 15 & 36 & \\
\hline N1 & 27 & 21 & \\
\hline N2 & 19 & 16 & 0.542 \\
\hline N3a & 32 & 18 & \\
\hline N3b & 23 & 5 & \\
\hline M0 or M1 & & 109 \\
\hline M0 & 92 & 7 & \\
\hline M1 & 4 & & \\
\hline * High and medium differentiated tubular adenocarcinoma and papillary adenocarcinoma were \\
regarded as differentiated types; mucous adenocarcinoma, signet ring cell carcinoma, low and \\
undifferentiated adenocarcinoma were regarded as undifferentiated types.
\end{tabular}

\section{Screening of the experimental cell lines and knockdown virus transfection}

We detected the STEAP1 mRNA level in GES-1, AGS, SGC-7901, and MGC-803 cells using RT-PCR (Fig. 1g). The results showed that STEAP1 was more highly expressed in SGC-7901 and MGC-803 cells. The western blot analysis yielded the same conclusion (Fig. 1h). Therefore, we selected SGC-7901 and MGC-803 cells as experimental cell lines. We overexpressed and knocked down the STEAP1 gene by transfecting the STEAP1 plasmid and STEAP1 shRNA. We transfected negative control (NC) virus and three kinds of STEAP1-shRNAs into SGC-7901 cells and detected the knockdown efficiency on mRNA and protein levels using RT-PCR and western blot analysis. The results showed that shRNA2 had the highest knockdown efficiency (Fig. 1i). Subsequently, we transfected NC virus and STEAP1-shRNA2 into SGC7901 and MGC-803 cells. The results showed successful transfection and knockdown using RT-PCR and western blot analysis (Fig. 1j-k).

STEAP1 gene regulates the cell cycle via the Akt/Fox01 pathway to influence cell proliferation. 
The CCK-8 assay results showed that the absorbance in the sh-STEAP1 group was lower than that in the NC group at 48 h, 72 h, and $96 \mathrm{~h}$ in both the SGC-7901 and MGC-803 cell lines (Fig. 2a). In another group of comparisons, we found that the absorbance of the STEAP1 plasmid vector group was higher than that of the NC group at 24 h, 48 h, 72 h, and $96 \mathrm{~h}$ in both the SGC-7901 and MGC-803 cell lines (Fig. 2b). The CCK-8 results indicated that the STEAP1 gene can influence cell proliferation. In the colony formation assay, we found that when STEAP1 was knocked down, the colony number was lower than that in the NC group (Fig. 2C), while when STEAP1 was overexpressed, the colony number was higher than that in the empty vector group (Fig. 2d). The colony formation assay results also indicated that the STEAP1 gene can influence cell proliferation. Next, we used flow cytometry to detect the cell cycle. The results showed that when STEAP1 was knocked down, the percentage of the cells in S phase was decreased, and the percentages of cells in G0/G1 and G2/M phase was increased in both SGC-7901 and MGC-803 cells (Fig. 2e). When STEAP1 was overexpressed, the percentage of $S$ phase cells was increased, and the percentages of G0/G1 and G2/M phase cells was decreased in both SGC-7901 and MGC-803 cells (Fig. 2f). The flow cytometry assay indicated that the STEAP1 gene can influence cell proliferation by influencing the cell cycle. Finally, we detected cell cycle related proteins and pathway proteins to identify the underlying mechanism. The Western blot analysis showed that when STEAP1 was down regulated, CDK4 and Cyclin D1 were relatively down regulated, and P27 was upregulated. Total AKT (AKT) and total Fox01 (Fox01) showed no significant change, while phosphorylated AKT (P-AKT) was down regulated, and phosphorylated FoxO1 (P-Fox01) was upregulated (Fig. 2g). When STEAP1 was upregulated, CDK4 and Cyclin D1 were relatively upregulated, and P27 was down regulated. AKT and FoxO1 also showed no significant change, while P-AKT was upregulated and P-Fox01 was down regulated (Fig. 2h).

\section{STEAP1 regulates cell migration and invasion via EMT.}

The transwell assay results showed that the number of migrating cells in the sh-STEAP1 group was lower than that in the NC group in both SGC-7901 and MGC-803 cells (Fig. 3a). The number of migrating cells in the STEAP1 plasmid group was higher than that in the empty vector group (Fig. 3b). The results of the wound healing assay also provided consistent conclusions. In SGC-7901 cells, the migration distance between $0 \mathrm{~h}$ and $96 \mathrm{~h}$ in the NC group was more obvious than that in the sh-STEAP1 group. The migration distance of the empty vector group was shorter than that of the STEAP1 plasmid group (Fig. 3c). This result was also identified in MGC-803 cells. The migration distance of the NC group was longer than that of the sh-STEAP1 group, and the migration distance of the empty vector group was shorter than that of the STEAP1 plasmid group (Fig. 3d). The results of the above two experiments indicated that when STEAP1 was knocked down, the cell migration ability was decreased, whereas it increased after the overexpression of STEAP1. We also used a transwell assay and placed Matrigel into the upper chamber for detecting the effect of the STEAP1 gene on cell invasion. The results showed that the number of invading cells in the sh-STEAP1 group was lower than that in the NC group in both SGC7901 and MGC-803 cells (Fig. 3e). The number of invading cells in the STEAP1 plasmid group was higher than that in the empty vector group (Fig. 3f). These results indicated that the STEAP1 gene had an effect on cell invasion. When STEAP1 was knocked down, the cell invasion ability was decreased and increased after overexpressing STEAP1. Finally, we detected cell migration- and invasion-related proteins using 
western blot analysis. The results showed that Vimentin, N-cadherin, MMP-2, and MMP-9 were downregulated and E-cadherin was upregulated after we downregulated the gene STEAP1 (Fig. 3g). In contrast, when STEAP1 was overexpressed, Vimentin, N-cadherin, MMP-2, and MMP-9 were relatively upregulated, and E-cadherin was downregulated (Fig. 3h).

\section{In vivo animal experiments}

Twelve BALB/c nude mice were used to study tumor formation and were randomly divided into two groups. We subcutaneously injected $3 \times 10^{6}$ of SGC-7901 NC cells per mouse in the first group, which was called the NC group. The mice in the other group were subcutaneously injected with $3 \times 10^{6}$ of SGC-7901 and sh-STEAP1 cells per mouse, which was called sh-STEAP1 group. The tumor sizes were measured every 2 days from 4 to 14 days after the injection and the results are shown in Fig. 4c. Twelve tumor specimens were removed from the mice on the 14th day. The results showed that the tumor size in the NC group was larger than that in the sh-STEAP1 group (Fig. 4a). Then, we carried out paraffin embedding, sectioning and IHC experiments with the tumor tissue. The results showed that the expression of Ki67 in the NC group was higher than that in the sh-STEAP1 group, while cleaved caspase-3 expression was lower than that in the sh-STEAP1 group (Fig. 4b). In the intraperitoneal tumorigenesis experiment, similar to the subcutaneous tumorigenesis experiment, twelve mice were divided into the NC and sh-STEAP1 groups, and $3 \times 10^{6}$ cells per mouse were injected by intraperitoneal injection. Three weeks later, the mice were sacrificed to observe the number of intraperitoneal tumors,including the mesentery, on the wall of the intestine (Fig. 4d). We used hemostatic forceps to clamp the two sides of the intestine to expand the mesentery,We found a huge number of tumors in the mesentery arranged like beads. However, tumors were rare or absent in the NC group (Fig. 4e). The number of tumors in the abdominal cavity of the two groups were statistically analyzed and the difference was statistically significant (Fig. 4f). We enlarged the image of a case in NC group when the abdominal cavity of nude mice was just opened. We can clearly see that the tumors covered the abdominal cavity, and several larger tumors on the mesenteric and intestinal wall were marked at the arrow. (Fig. $4 \mathrm{~g}$ )

\section{Discussion}

As a global health problem, cancer affects the quality of patients' lives worldwide and causes thousands of deaths every year[1]. STEAP1 is overexpressed in many kinds of cancers, such as prostate cancer, colon cancer, bladder cancer, ovarian cancer, pancreatic cancer, testicular cancer, breast cancer, cervical cancer and Ewing sarcoma[10, 12]. In our study, STEAP1 was overexpressed in gastric cancer and closely related to the prognosis of patients (Fig. 1b). The 5-year OS of patients with a low expression of STEAP1 was $60.7 \%$, while that of patients with a high expression of STEAP1 was only $25.9 \%$ (Fig. 1d). STEAP1 plays a role as an oncogene in gastric cancer, and this result was consistent with the conclusion that STEAP1 is an oncogene in other kinds of cancer. Tumor cell growth, metastasis, proliferation, migration and invasion are basic biological functions[22]. We downregulated or upregulated STEAP1 by using lentivirus knockdown or STEAP1 plasmids, respectively, to detect the changes in the above functions. The 
results of the CCK-8 and colony formation assays indicated that when we overexpressed STEAP1, the percentage of cells in the $S$ phase increased, that in the G0/G1 and G2/M phases decreased, and the cell proliferation ability was also improved. In contrast, when STEAP1 was knocked down, the percentage of cells in the S phase decreased, that in the G0/G1 and G2/M phases increased, and cell proliferation was reduced (Fig. 2a-f). Cyclin D1 is known as an oncogene and overexpressed in many kinds of cancers[23]. By binding with CDK4 ( a partner kinases of cyclin D1), cyclin D1 can release transcription factors and advance cell cycle progression from the $\mathrm{G} 1$ phase to the S phase. The P27 protein limits cell cycle progression, mainly by inhibiting complex formation, such as CyclinD1-CDK4 and CyclinE-CDK2, to block the cell cycle in the G1 phase. Previous studies have found that the AKT pathway is one of the main signaling pathways influencing cancer cell proliferation[24-29]. Therefore, it was reasonable to consider that STEAP1 can affect cell proliferation via the AKT pathway. Our results also showed that when STEAP1 was downregulated, P-AKT, CDK4 and Cyclin D1 were relatively downregulated, and P27 and PFox01 were upregulated (Fig. 2g). When STEAP1 was upregulated, P-AKT, CDK4 and Cyclin D1 were relatively upregulated, and P-FoxO1 and P27 were downregulated. P-AKT was upregulated and P-Fox01 was downregulated (Fig. 2h). These results indicated that STEAP1 can regulate the cell cycle via the Akt/Fox01 pathway to influence cell proliferation. The results of the transwell and wound healing assays showed that when we overexpressed STEAP1, cell migration and invasion increased. In contrast, when STEAP1 was knocked down, the two abilities above decreased (Fig. 3a-f). Next, EMT-related proteins, MMP2 and MMP9 were detected by western blotting. The EMT-related proteins include N-cadherin ,vimentin and E-cadherin. A previous study showed that when cells tend to migrate and metastasize,the protein expression of vimentin and $\mathrm{N}$-cadherin increases and that of E-cadherin decreases[30, 31]. In addition, many studies have identified that MMP2 and MMP9 are closely related to tumor migration and invasion and explained the mechanism[32-34]. The results of our study showed that when STEAP1 was overexpressed, N-cadherin, Vimentin, MMP-2 and MMP-9 were relatively upregulated, and E-cadherin was downregulated. When STEAP1 was downregulated, N-cadherin, MMP-9 and MMP-2 were downregulated, and E-cadherin was upregulated (Fig. $3 \mathrm{~h}-\mathrm{g}$ ). These results indicated that the STEAP1 gene may regulate cell migration and invasion via EMT. The result of the apoptosis marker cleaved caspase- 3 and proliferation-related nuclear antigen $\mathrm{Ki}-67$ by IHC assay also indicated that STEAP1 plays a very important role in cell proliferation in vitro. Until the early 1990s, We always admitted that peritoneal metastasis of gastric cancer is a kind of terminal disease and the effect of systemic chemotherapy is limited for it. In our study, we established a peritoneal metastasis model by intraperitoneal injection of tumor cells into mice. The results showed that the number of tumors on the mesentery in the NC group was higher than that in the sh-STEAP1 group. Through this experiment, we verified the effect of STEAP1 on tumor cell invasion and metastasis in vivo.

\section{Conclusions}

In conclusion, STEAP1 was overexpressed in gastric cancer and closely connected with OS. STEAP1 can regulate the cell cycle via the Akt/Fox01 pathway to influence cell proliferation. STEAP1 may affect cell migration and invasion via EMT induction. 


\section{Declarations}

\section{Ethics approval and consent to participate}

All patients were approved by the ethics committee of China Medical University to participate in the study and provided written informed consent. All animal experimental steps were approved by the Animal Research Committee of China Medical University.

\section{Consent for publication}

Not applicable

\section{Availability of data and materials}

Not applicable

\section{Competing interests}

There are no competing interests to declare

\section{Funding}

This work was supported by National Natural Science Foundation of China (No.81602522).

\section{Author contributions}

Project design: Hui-mian Xu

Clinical data collection囚Zhe Zhang

Cell function experiment囚Zhe Zhang

Animal experiment囚Zhe Zhang

Data statistics and analysis $₫$ Zhe Zhang

Manuscript writing and submissionखZhe Zhang

Acknowledgements

We would like to thank Editage (www.editage.com) for English language editing.

\section{References}

1. Pisani P, Parkin DM, Bray F, Ferlay J. Estimates of the worldwide mortality from 25 cancers in 1990. Int J Cancer. 1999;83(1):18-29. 
2. Berardi R, Scartozzi M, Romagnoli E, Antognoli S, Cascinu S. Gastric cancer treatment: a systematic review. Oncol Rep. 2004;11(4):911-6.

3. Zong $L$, Abe M, Seto Y, Ji J. The challenge of screening for early gastric cancer in China. Lancet. 2016;388(10060):2606.

4. Jia B, Liu H, Kong Q, Li B. RKIP expression associated with gastric cancer cell invasion and metastasis. Tumour Biol. 2012;33(4):919-25.

5. Mayer B, Funke I, Johnson JP. High expression of a Lewis(x)-related epitope in gastric carcinomas indicates metastatic potential and poor prognosis. Gastroenterology. 1996;111(6):1433-46.

6. Jia Y, Dong B, Tang L, Liu Y, Du H, Yuan P, et al. Apoptosis index correlates with chemotherapy efficacy and predicts the survival of patients with gastric cancer. Tumour Biol. 2012;33(4):1151-8.

7. Oishi Y, Watanabe Y, Yoshida Y, Sato Y, Hiraishi T, Oikawa R, et al. Hypermethylation of Sox17 gene is useful as a molecular diagnostic application in early gastric cancer. Tumour Biol. 2012;33(2):38393.

8. Janjigian YY, Werner D, Pauligk C, Steinmetz K, Kelsen DP, Jager E, et al. Prognosis of metastatic gastric and gastroesophageal junction cancer by HER2 status: a European and USA International collaborative analysis. Ann Oncol. 2012;23(10):2656-62.

9. Yu J, Cheng YY, Tao Q, Cheung KF, Lam CN, Geng H, et al. Methylation of protocadherin 10, a novel tumor suppressor, is associated with poor prognosis in patients with gastric cancer. Gastroenterology. 2009;136(2):640-51. e1.

10. Hubert RS, Vivanco I, Chen E, Rastegar S, Leong K, Mitchell SC, et al. STEAP: a prostate-specific cellsurface antigen highly expressed in human prostate tumors. Proc Natl Acad Sci U S A 1999 Dec 7;96(25):14523-8.

11. Yang D, Holt GE, Velders MP, Kwon ED, Kast WM. Murine six-transmembrane epithelial antigen of the prostate, prostate stem cell antigen, and prostate-specific membrane antigen: prostate-specific cellsurface antigens highly expressed in prostate cancer of transgenic adenocarcinoma mouse prostate mice. Cancer Res. 2001 Aug 1;61(15):5857-60.

12. Korkmaz KS, Elbi C, Korkmaz CG, Loda M, Hager GL, Saatcioglu F. Molecular cloning and characterization of STAMP1, a highly prostate-specific six transmembrane protein that is overexpressed in prostate cancer. J Biol Chem. 2002;277(39):36689-96.

13. Gomes IM, Maia CJ, Santos CR. STEAP proteins: from structure to applications in cancer therapy. Mol Cancer Res. 2012 May;10(5):573-87.

14. Challita-Eid PM, Morrison K, Etessami S, An Z, Morrison KJ, Perez-Villar JJ, et al Monoclonal antibodies to six-transmembrane epithelial antigen of the prostate- 1 inhibit intercellular communication in vitro and growth of human tumor xenografts in vivo. Cancer Res. 2007 Jun 15;67(12):5798-805.

15. Kobayashi H, Nagato T, Sato K, Aoki N, Kimura S, Murakami M, et al. Recognition of prostate and melanoma tumor cells by six-transmembrane epithelial antigen of prostate-specific helper T 
lymphocytes in a human leukocyte antigen class II-restricted manner. Cancer Res. 2007 Jun 1;67(11):5498-504.

16. Gomes IM, Arinto P, Lopes C, Santos CR, Maia CJ. STEAP1 is overexpressed in prostate cancer and prostatic intraepithelial neoplasia lesions, and it is positively associated with Gleason score. Urol Oncol. 2014 Jan;32(1):53.e23-9.

17. Maia CJ, Socorro S, Schmitt F, Santos CR. STEAP1 is over-expressed in breast cancer and downregulated by 17beta-estradiol in MCF-7 cells and in the rat mammary gland. Endocrine. 2008;34(13):108-16.

18. Cheung IY, Feng Y, Danis K, Shukla N, Meyers P, Ladanyi M, et al. Novel markers of subclinical disease for Ewing family tumors from gene expression profiling. Clin Cancer Res. 2007 Dec 1;13(23):6978-83.

19. Hayashi T, Oue N, Sakamoto N, Anami K, Oo HZ, Sentani K, et al. Identification of transmembrane protein in prostate cancer by the Escherichia coli ampicillin secretion trap: expression of CDON is involved in tumor cell growth and invasion. Pathobiology. 2011;78(5):277-84.

20. Moreaux J, Kassambara A, Hose D, Klein B. STEAP1 is overexpressed in cancers: a promising therapeutic target. Biochem Biophys Res Commun. 2012 Dec 14;429(3-4):148-55.

21. Lee CH, Chen SL, Sung WW, Lai HW, Hsieh MJ, Yen HH, et al. The Prognostic Role of STEAP1 Expression Determined via Immunohistochemistry Staining in Predicting Prognosis of Primary Colorectal Cancer: A Survival Analysis. Int J Mol Sci. 2016 Apr;17(4):592.

22. Yang $Y$, Xun N, Wu JG. Long non-coding RNA FGF14-AS2 represses proliferation, migration, invasion, and induces apoptosis in breast cancer by sponging miR-205-5p. Eur Rev Med Pharmacol Sci. 2019 Aug;23(16):6971-82.

23. Deep G, Singh RP, Agarwal C, Kroll DJ, Agarwal R. Silymarin and silibinin cause G1 and G2-M cell cycle arrest via distinct circuitries in human prostate cancer PC3 cells: a comparison of flavanone silibinin with flavanolignan mixture silymarin. Oncogene. 2006 Feb 16;25(7):1053-69.

24. Song Q, Qin S, Pascal LE, Zou C, Wang W, Tong H, et al. SIRPB1 promotes prostate cancer cell proliferation via Akt activation. Prostate. 2020 Mar;80(4):352-64.

25. Chen Q, Gao Y, Yu Q, Tang F, Zhao PW, eta I. miR-30a-3p inhibits the proliferation of liver cancer cells by targeting DNMT3a through the PI3K/AKT signaling pathway. Oncol Lett. 2020;19(1):606 - 14.

26. Yu H, Yao J, Du M, Ye J, He X, Yin L. CDKN3 promotes cell proliferation, invasion and migration by activating the AKT signaling pathway in esophageal squamous cell carcinoma. Oncol Lett. 2020;19(1):542-8.

27. Hou B, Li W, Li J, Ma J, Xia P, Liu Z, et al. Tumor suppressor LHPP regulates the proliferation of colorectal cancer cells via the PI3K/AKT pathway. Oncol Rep. 2020 Feb;43(2):536-48.

28. Yun SH, Han SH, Park JI. COUP-TFII Knock-down Promotes Proliferation and Invasion in Colorectal Cancer Cells via Activation of Akt Pathway and Up-regulation of FOXC1. Anticancer Res. 2020;40(1):177-90. 
29. Si X, Xu F, Xu F, Wei M, Ge Y, Chenge S. CADM1 inhibits ovarian cancer cell proliferation and migration by potentially regulating the PI3K/Akt/mTOR pathway. Biomed Pharmacother. 2019;123:109717.

30. Santamaria PG, Moreno-Bueno G, Portillo F, Cano A. EMT: Present and future in clinical oncology. Mol Oncol. 2017;11(7):718-38.

31. De Seta D, Mancini P, Minni A, Prosperini L, De Seta E, Attanasio G, et al. Bell's palsy: symptoms preceding and accompanying the facial paresis. ScientificWorldJournal. 2014;2014:801971.

32. Buccione R, Caldieri G, Ayala I. Invadopodia: specialized tumor cell structures for the focal degradation of the extracellular matrix. Cancer Metast Rev. 2009;28(1-2):137-49.

33. Egeblad M, Werb Z. New functions for the matrix metalloproteinases in cancer progression. Nat Rev Cancer. 2002;2(3):161-74.

34. Ghosh S, Basu M, Roy SS. ETS-1 protein regulates vascular endothelial growth factor-induced matrix metalloproteinase-9 and matrix metalloproteinase-13 expression in human ovarian carcinoma cell line SKOV-3. J Biol Chem. 2012;287(18):15001-15.

35. Liang Y, Xing X, Beamer MA, Swindell WR, Sarkar MK, et al. Six-transmembrane epithelial antigens of the prostate comprise a novel inflammatory nexus in patients with pustular skin disorders. J Allergy Clin Immunol. 2017;139(4):1217-27.

36. Yonemura $Y$, Ishibashi H, Hirano M, Mizumoto A, Takeshita K, Noguchi K, et al. Effects of Neoadjuvant Laparoscopic Hyperthermic Intraperitoneal Chemotherapy and Neoadjuvant Intraperitoneal/Systemic Chemotherapy on Peritoneal Metastases from Gastric Cancer. Ann Surg Oncol. 2017;24(2):478-85.

\section{Figures}




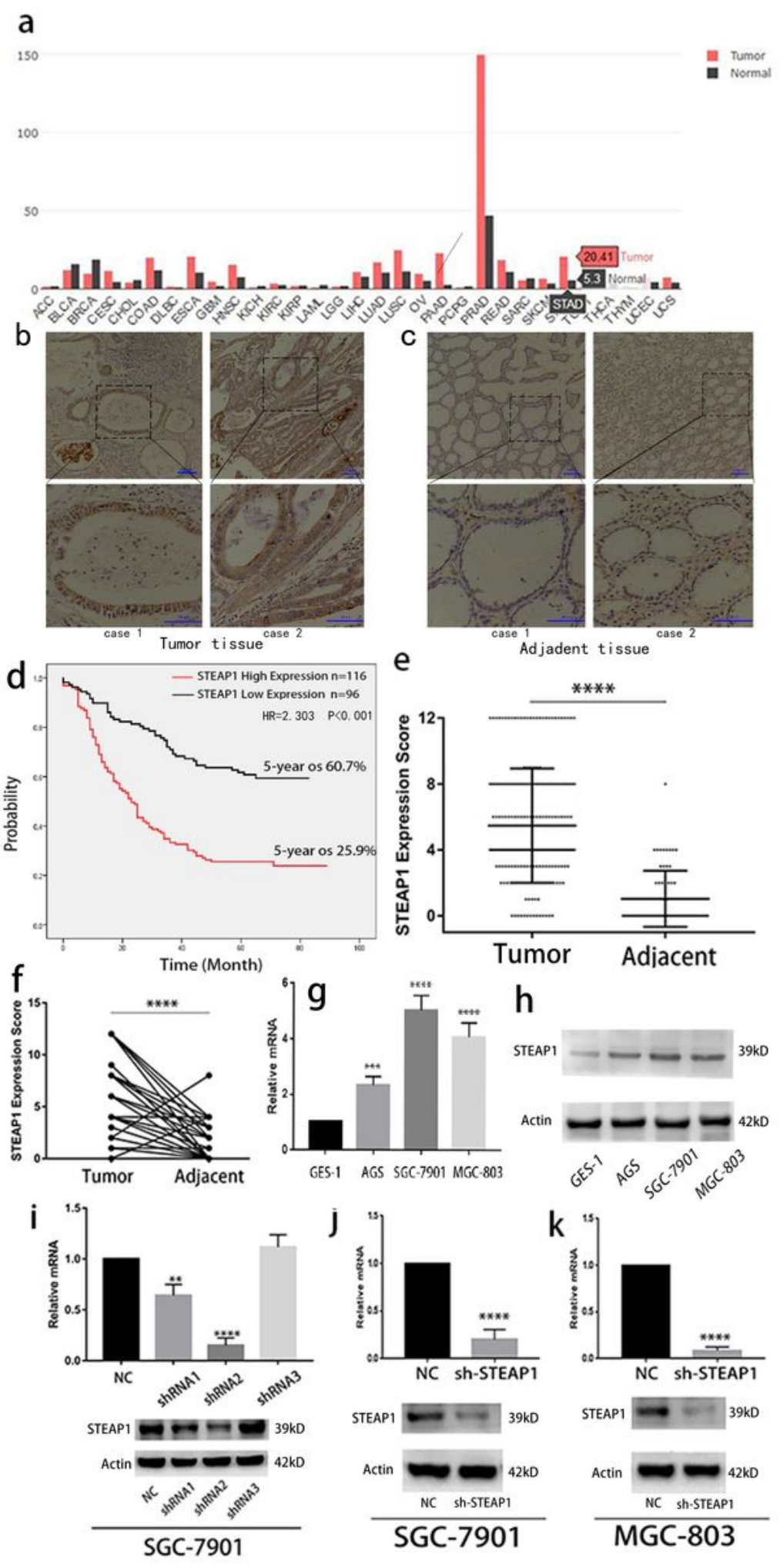

\section{Figure 1}

STEAP1 is highly expressed in gastric cancer and closely associated with OS; and STEAP1 lentivirus was successfully transfected into the cell line. (a) The GEPIA database shows the expression of STEAP1 in various types of cancer. (b) IHC assay shows the expression of STEAP1 protein in gastric cancer tissues (100x and 400x). (c) IHC assay shows the expression of STEAP1 protein in gastric paracancerous tissues (100x and 400x). (d) Kaplan-Meier analysis was used to show the overall survival rates in gastric cancer 
patients with high and low expression levels of STEAP1. (e) The staining score distribution of 212 cases of gastric cancer and 60 cases of paracancerous tissues. $(\mathrm{f})$ The staining score distribution of 60 matched tissues. (g) Detection of the expression of STEAP1 RNA expression levels in GES-1, AGS, SGC7901 and MGC-803 cells. (h) Detection of the protein expression levels of STEAP1 in GES-1, AGS, SGC7901 and MGC-803 cells. (i) Detection of the knockdown efficiency of three lentiviruses at the mRNA and protein levels using RT-PCR and Western blot analysis, respectively. (j) Detection of STEAP1 knockdown efficiency in the SGC-7901 cell line. (k) Detection of STEAP1 knockdown efficiency in the MGC-803 cell line. OS, overall survival. $\left({ }^{\star} p<0.05,{ }^{* \star} p<0.01,{ }^{* \star *} p<0.001,{ }^{\star \star * \star *} p<0.0001\right)$ 
a

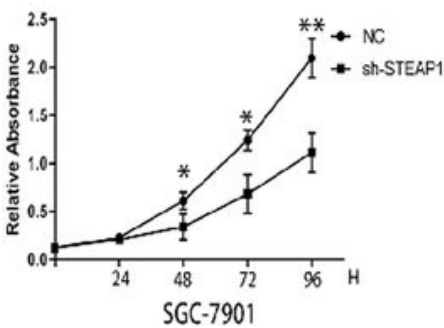

b
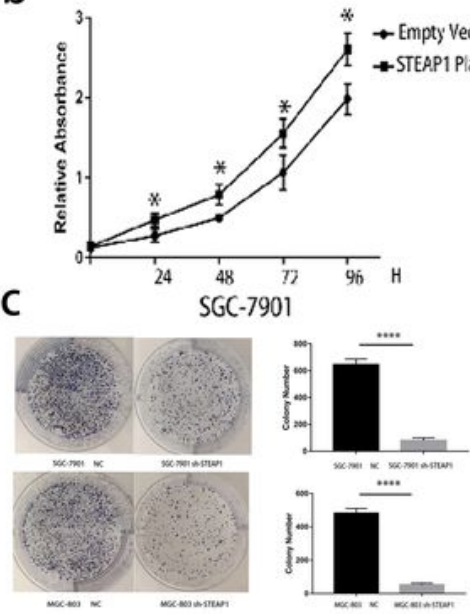

e
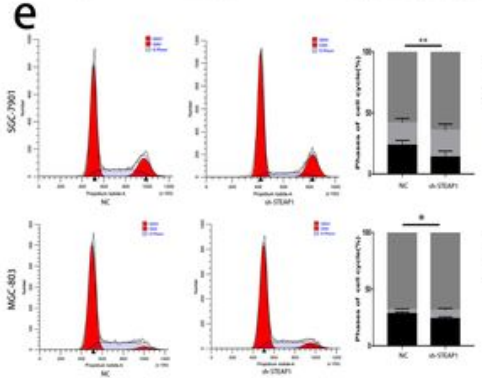

g

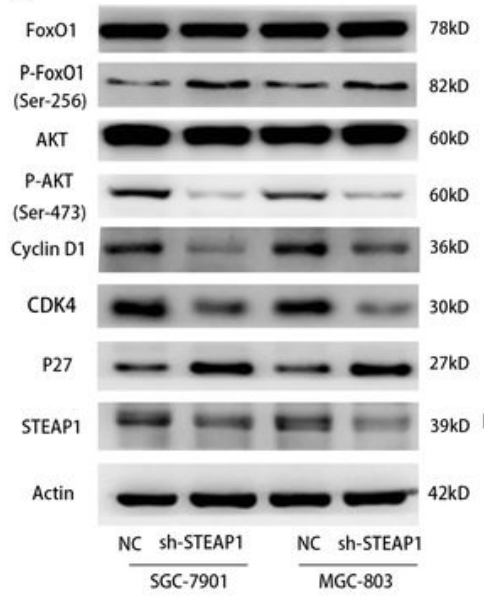

Figure 2
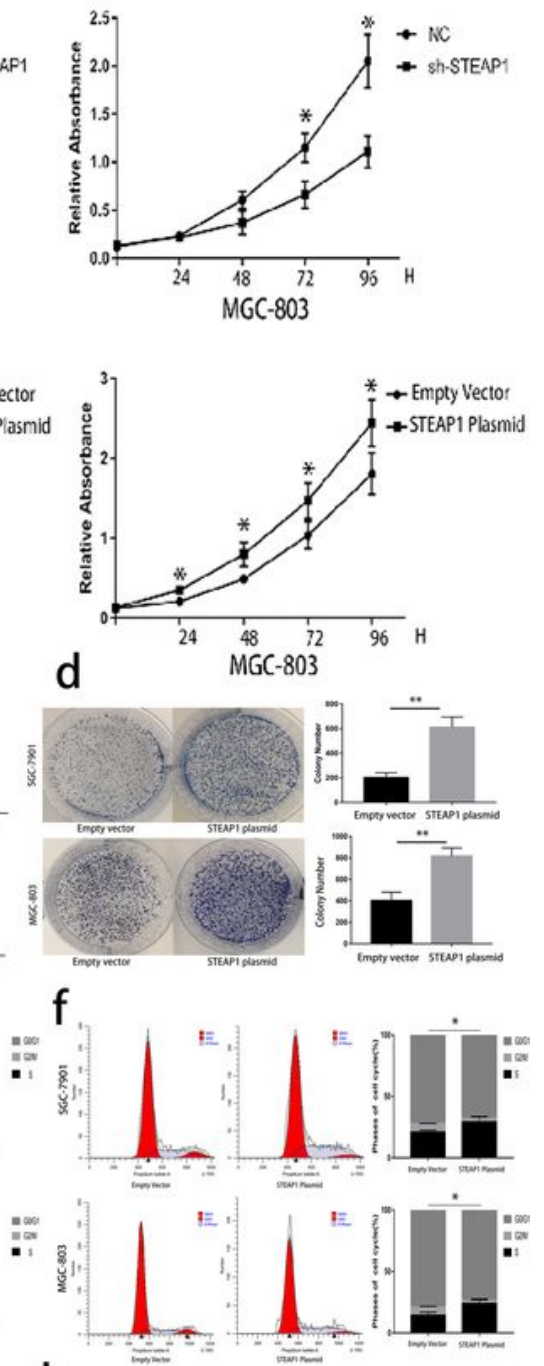

h

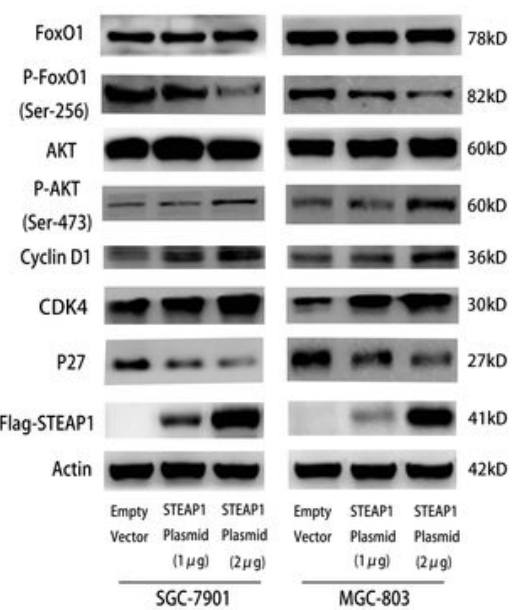

Functional experiments related to cell proliferation in the SGC-7901 and MGC-803 cell lines. (a) CCK-8 assay showed the differences in cell proliferation before and after knocking down STEAP1 in the SGC7901 and MGC-803 cell lines. (b) CCK-8 assay showed the differences in cell proliferation before and after overexpression of STEAP1 in the SGC-7901 and MGC-803 cell lines. (c) Colony formation assay showed the differences in cell proliferation before and after knocking down STEAP1 in the SGC-7901 and 
MGC-803 cell lines. (d) Colony formation assays showed the differences in cell proliferation before and after STEAP1 overexpression in the SGC-7901 and MGC-803 cell lines. (e) Flow cytometry detected the differences in cell cycle before and after knocking down STEAP1 in the SGC-7901 and MGC-803 cell lines. (f) Flow cytometry detected the difference in cell cycle before and after the overexpression of STEAP1 in the SGC-7901 and MGC-803 cell lines. (g) Western blot analysis detected the differences in protein expression related to the cell cycle and pathway before and after knocking down STEAP1 in the SGC7901 and MGC-803 cell lines. (h) Western blot analysis detected the differences in protein expression related to the cell cycle and pathway before and after the overexpression of STEAP1 in the SGC-7901 and MGC-803 cell lines. $\left({ }^{\star} p<0.05,{ }^{\star} \mathrm{p} p<0.01,{ }^{\star \star \star} \mathrm{p}<0.001,{ }^{\star \star \star \star *} \mathrm{p}<0.0001\right)$ 

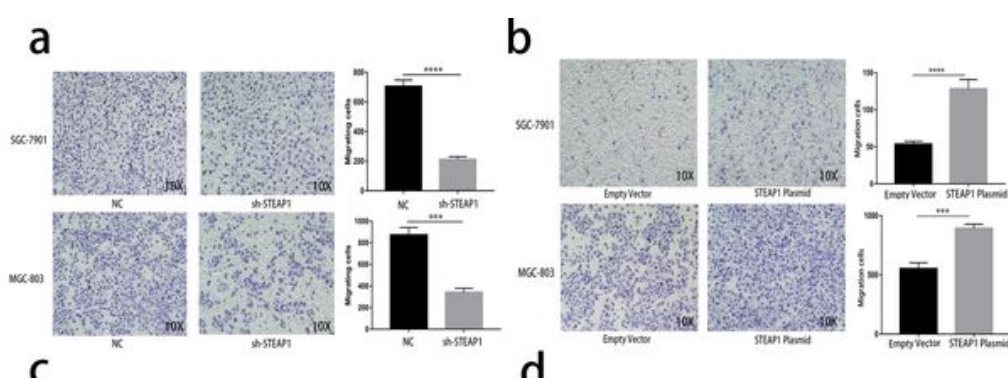

C

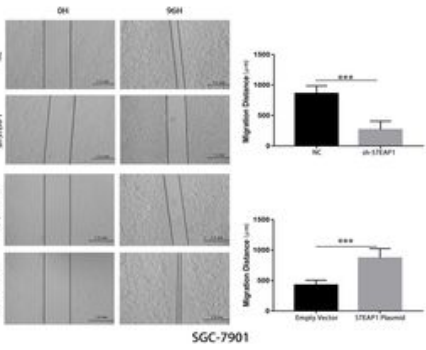

d
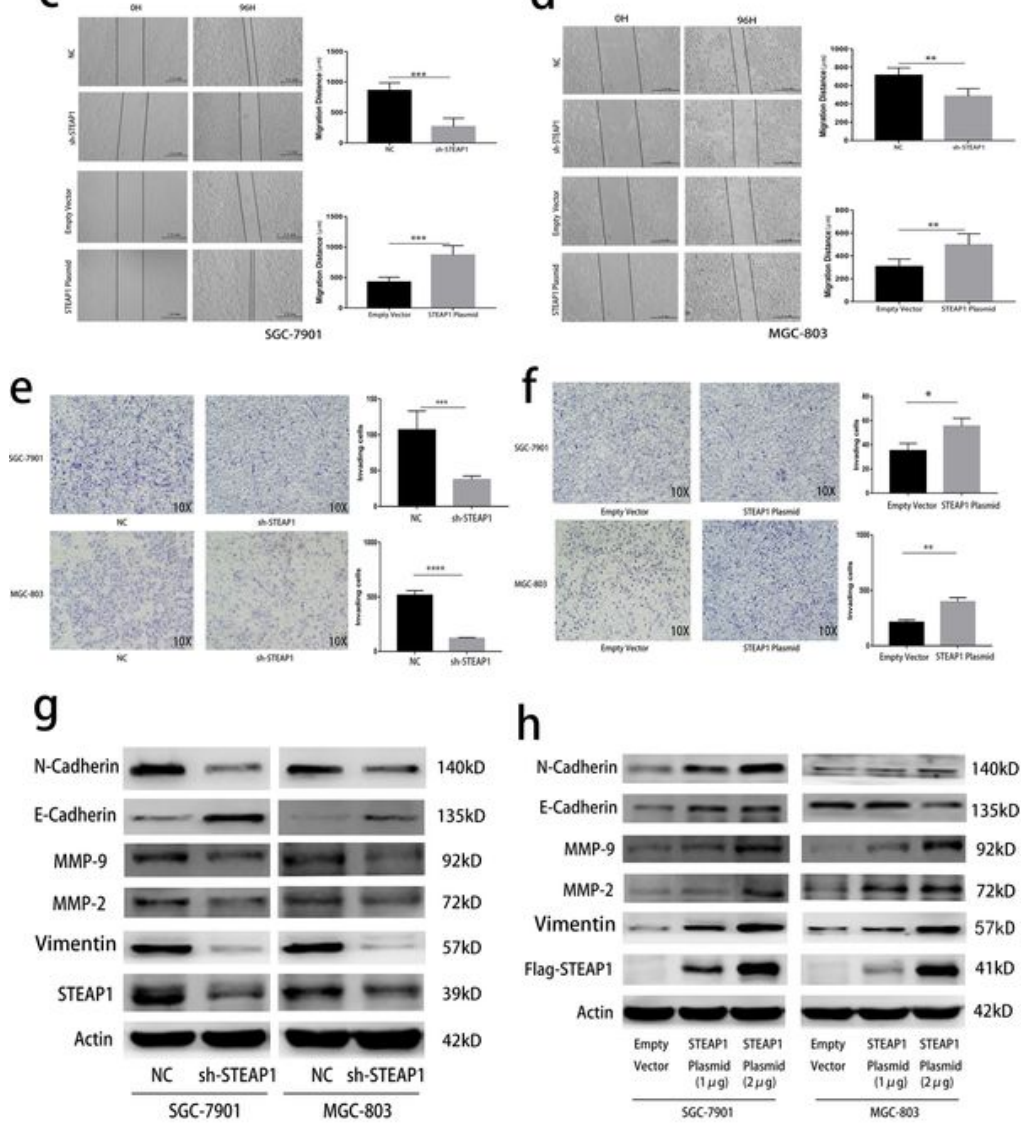

$\mathrm{h}$

N-Cadherin $=-\square=140 \mathrm{kD}$ E-Cadherin — - - - - $135 \mathrm{kD}$

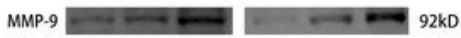
MMP.2 $-2+2072 \mathrm{kD}$ Vimentin - - - - $-57 \mathrm{kD}$ Flag-STEAP1 Actin $42 \mathrm{kD}$ EmpEY STEAPI STEAP1 Empty STEAP1 STEAPI

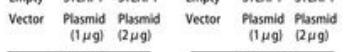
SGC-7901 MGC-803

\section{Figure 3}

Functional experiments related to cell migration and invasion in the SGC-7901 and MGC-803 cell lines. (a) Detection of cell migration differences before and after knocking down STEAP1 in the SGC-7901 and MGC-803 cell lines using transwell assay. (b) Detection of cell migration differences before and after the overexpression of STEAP1 in the SGC-7901 and MGC-803 cell lines by transwell assay. (c) Detection of cell migration differences before and after knocking down STEAP1 in the SGC-7901 and MGC-803 cell 
lines using wound healing assay. (d) Detection of cell migration differences before and after the overexpression of STEAP1 in the SGC-7901 and MGC-803 cell lines using wound healing assay. (e) Detection of cell invasion differences before and after knocking down STEAP1 in the SGC-7901 and MGC-803 cell lines using transwell assay. (f) Detection of cell invasion differences before and after the overexpression of STEAP1 in the SGC-7901 and MGC-803 cell lines using transwell assay. (g) Western blot analysis detected the difference in protein expression related to cell migration, invasion and EMT before and after knocking down STEAP1 in the SGC-7901 and MGC-803 cell lines. (h) Western blot analysis detected the difference in protein expression related to cell migration, invasion and EMT before and after the overexpression of STEAP1 in the SGC-7901 and MGC-803 cell lines. $\left({ }^{*} p<0.05,{ }^{\star \star} p<0.01\right.$, $\star \star \star * p<0.001, * \star \star * p<0.0001)$ 
a

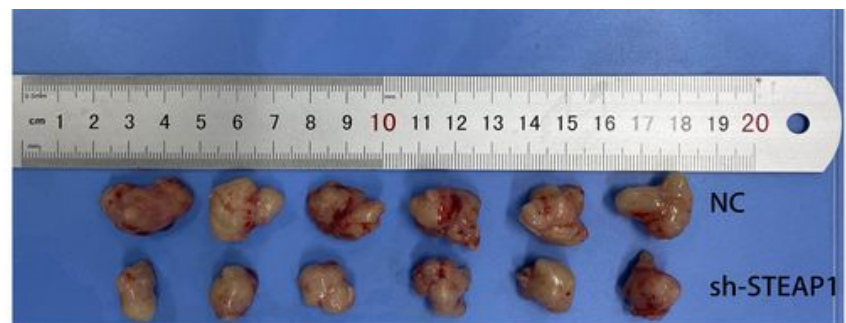

b
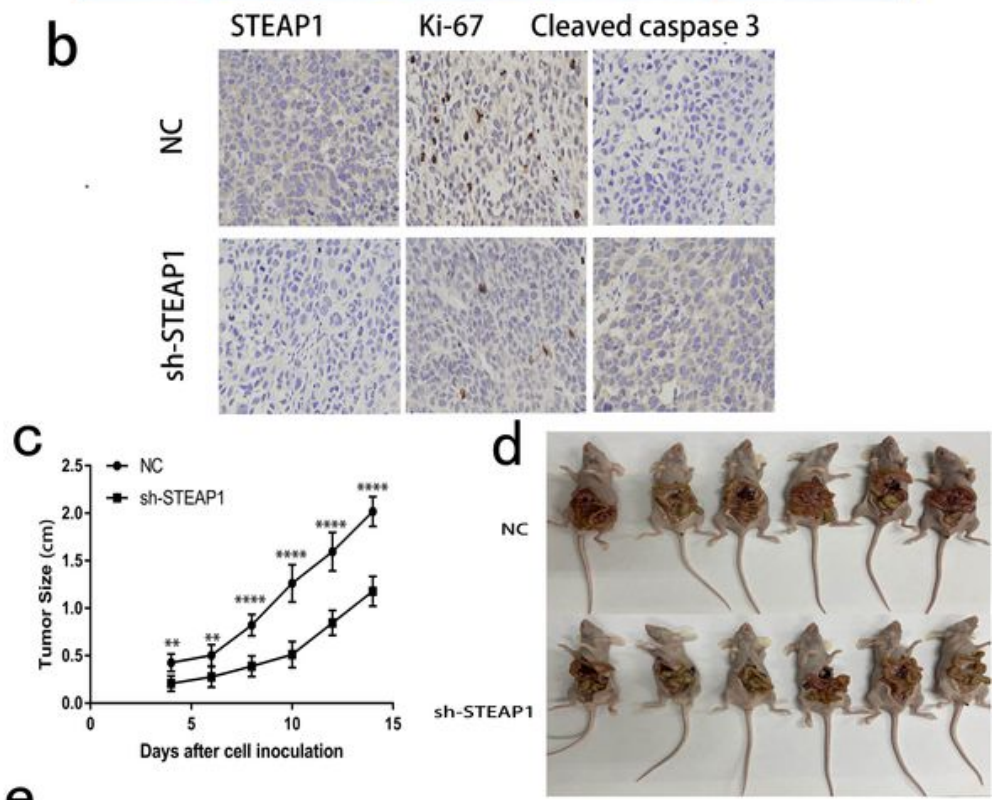

e
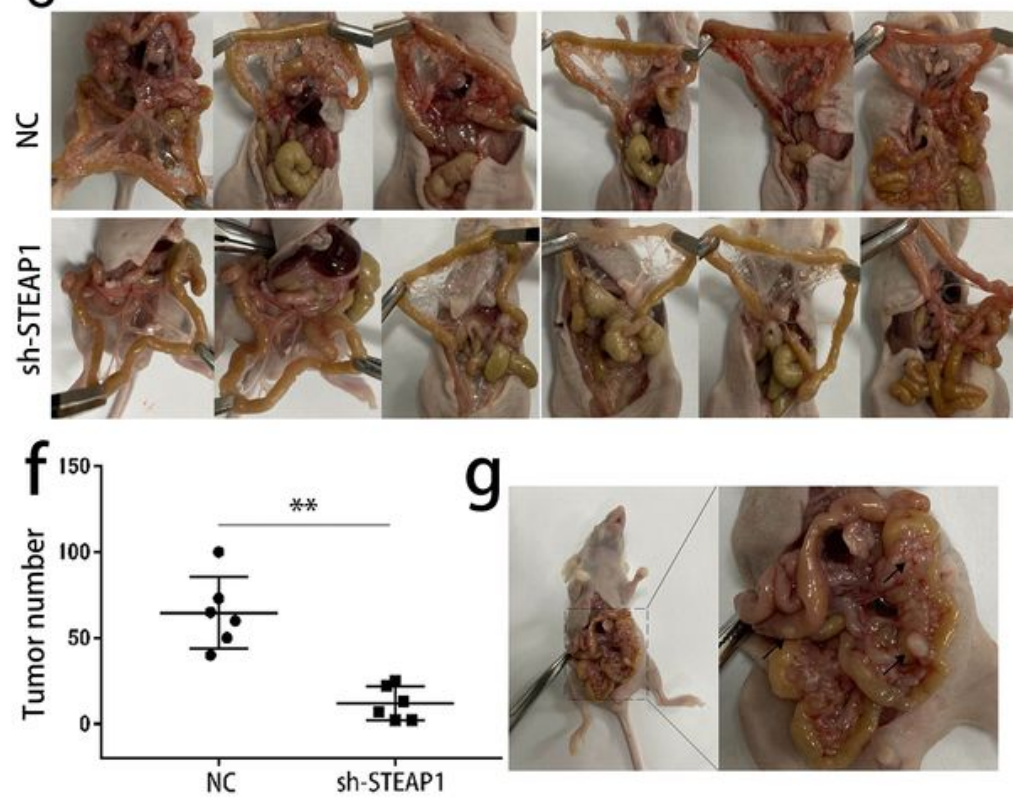

\section{Figure 4}

This figure shows the effect of the STEAP1 gene on cell proliferation and metastasis invivo through animal experiments. (a) The tumorigenesis experiment in nude mice shows the tumorsize differences between the SGC-7901 NC group and the sh-STEAP1 group. (b) IHC assayshows the expression of cell proliferation-related proteins in the tumors of nudemice (400x). (c) The difference in tumor size between the SGC-7901 NC group and sh-STEAP1 group in two weeks. (d) the mice were sacrificed to observe the 
number of intraperitoneal tumors,including the mesentery, on the wall of the intestine (e) On the whole, the number of mesenteric tumors in NC group was significantly larger than that in sh-STEAP1 group. (f) The tumor number difference between the SGC-7901 NC group and sh-STEAP1 group. (g) The tumors were found on the mesentery and the wall of the intestine,several larger tumors on the mesenteric and intestinal wall were marked at the arrow. . $\left.{ }^{\star} p<0.05,{ }^{* \star} p<0.01,{ }^{* \star *} p<0.001,{ }^{* \star *} p<0.0001\right)$. 